\title{
Jahrestagung 2017 der AOTrauma Deutschland
}

Michael J. Raschke, Philip Wilbrandt

Die Jahrestagung der AOTrauma Deutschland (AOTD) fand vom 12.-13.05.2017 in Münster statt. Im Mittelpunkt der Tagung stand die Strategie: Was ist die AO heute?

Zu dieser Diskussion kamen der Präsident, Klaus Renner, sowie weitere führende Vertreter der AO Foundation nach Deutschland.

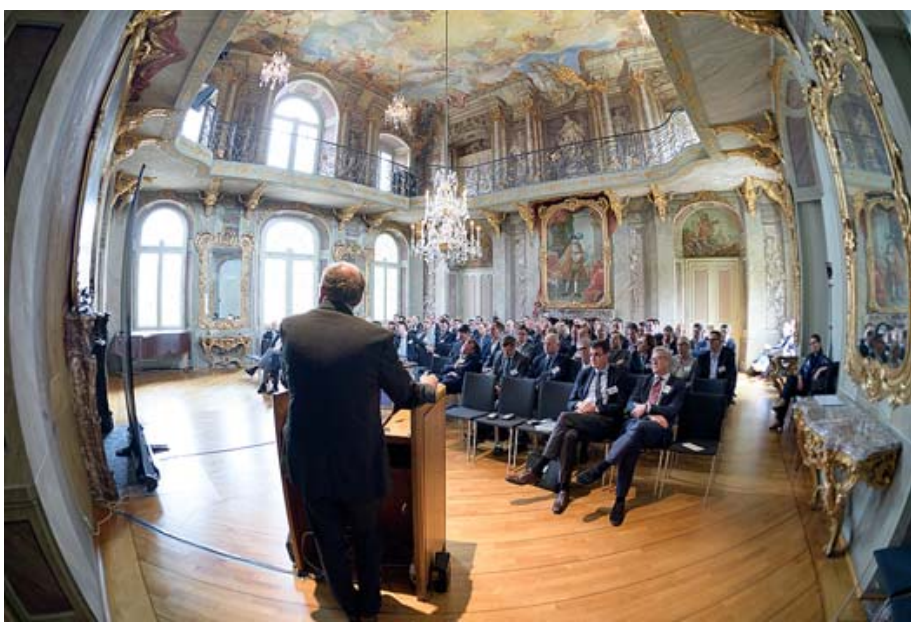

- Abb. 1 Eröffnung der Jahrestagung im Erbdrostenhof. Bild: AO Foundation.

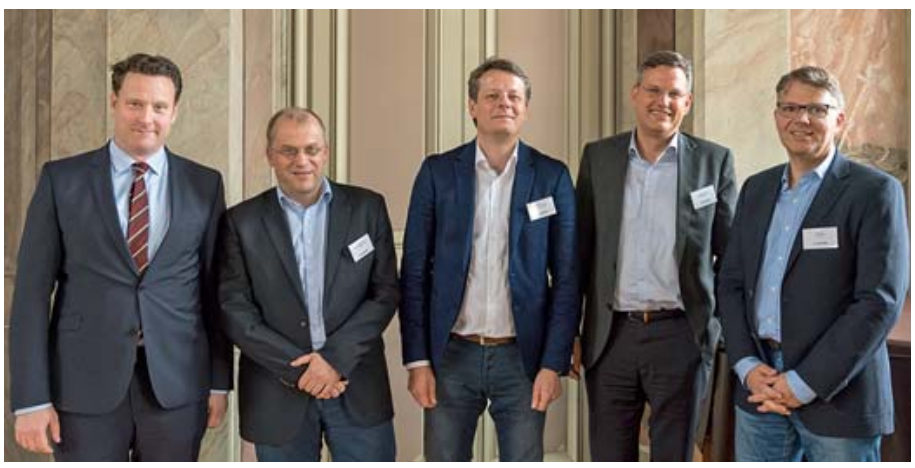

- Abb. 3 Lehmann, Schaser, Schiffer, Böcker und Mahlke. Bild: AO Foundation.

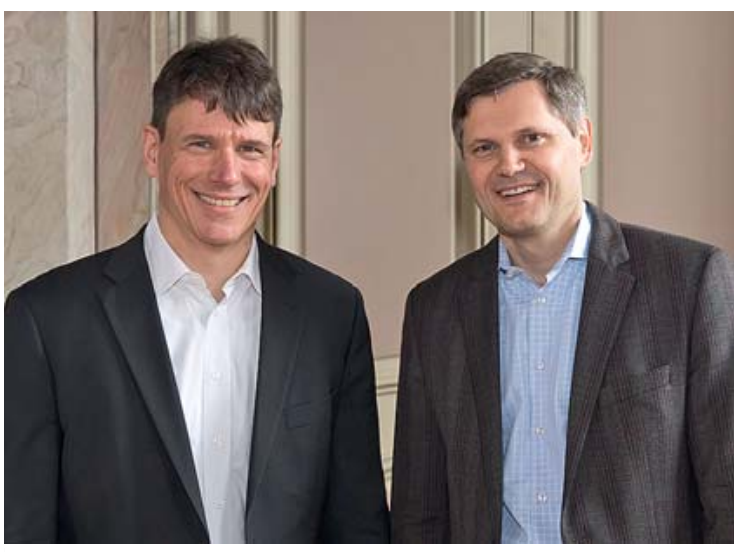

- Abb. 2 Georg Duda und Karl-Heinz Frosch nach ihrer Wahl. Bild: AO Foundation.

Die Jahrestagung wurde sehr unterhaltsam und geistreich vom Oberbürgermeister der Stadt Münster, Markus Lewe, eröffnet. Dieser begrüßte die 101 Mitglieder und 19 Gäste im Erbdrostenhof und hob die besondere Stellung der Klinik von Prof. Michael Raschke, dem Präsidenten der AOTD und Gastgeber der Jahrestagung hervor.

\section{Wahlen}

Auf der Mitgliederversammlung wurden Prof. Karl-Heinz Frosch (Hamburg) als Community Development Officer und Prof. Georg Duda (Berlin) als Research Officer für eine 2. Amtszeit wiedergewählt. Die Amtszeiten beginnen am 01.01.2018 und enden am 31.12.2020.

Im 12-köpfigen Beirat waren 7 Plätze neu zu vergeben. Diese werden ab dem 01.01.2018 besetzt von Wolfgang Böcker (München), Thomas Gösling (Braunschweig, Wiederwahl), Wolfgang Lehmann (Göttingen), Lutz Mahlke 
(Paderborn), Thomas Mückley (Erfurt, Wiederwahl), Klaus-Dieter Schaser (Dresden, Wiederwahl) und Gereon Schiffer (Bergisch-Gladbach). Die Amtszeit beginnt am 01.01.2018 und endet am 31.12.2020.

\section{Mitglied}

Auf der Jahrestagung wurde das 700. Mitglied, Prof. Andreas Eisenschenk (Berlin), begrüßt. Zum Zeitpunkt der Jahrestagung hatte die AOTrauma Deutschland 725 Mitglieder, ein Anstieg um 110 seit der letzten Jahrestagung.

\section{Ehrenmitglied}

Zum Ehrenmitglied wurde Prof. Dr. Dr. hc. Norbert P. Haas (Berlin) ernannt. Die Zeremonie erfolgte durch die Kiepenkerle.

\section{Reisestipendium}

Für das Reisestipendium 2017 gab es 10 Bewerbungen. Die Auswahlkommission, bestehend aus Prof. Karl-Heinz Frosch, Prof. Freddy Grützner und Prof. Wolfgang Lehmann entschied sich für Dr. Andreas Baranowski (Mainz), PD Dr. Arne Berner (Berlin), PD Dr. Max Kauther (Essen) und PD Dr. Gregor Stein (Köln). Alle erhalten ein Stipendium über jeweils 4000 EUR.

\section{AO unterstützte Klinik in Nepal}

Am 25.04.2015 zerstörte ein Erdbeben in Nepal beträchtliche Teile der medizinischen Infrastruktur. Seitdem unterstützt die AOTD Prof. Heiner Winker (Erfurt), der beim Aufbau des Dhulikhel Krankenhauses, Universitätsklinikum von Kathmandu, tatkräftig mithilft. Nach einem Vortrag über den Status quo überreichte Prof. Michael Raschke einen Scheck über weitere 5000 EUR an Prof. Heiner Winker für das Krankenhaus.

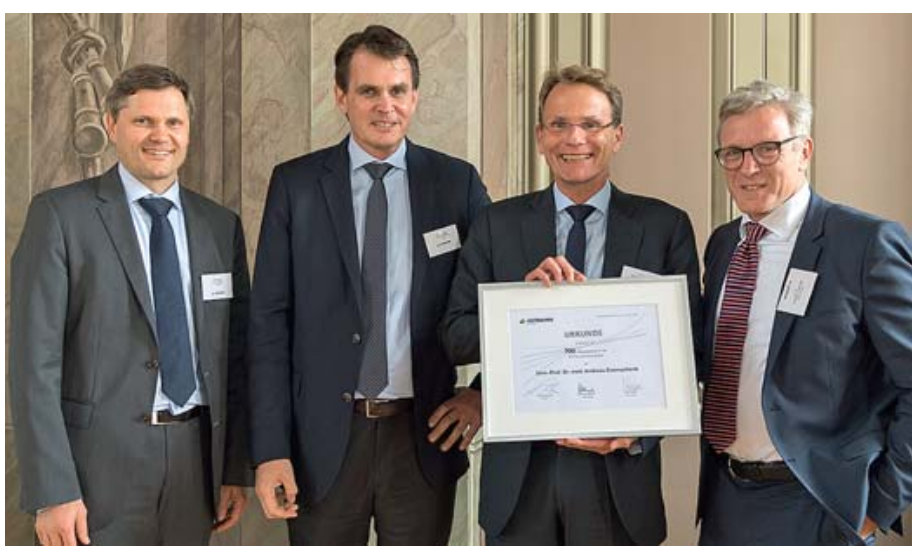

Abb. 4 Frosch, Stöckle, Eisenschenk und Raschke. Bild: AO Foundation.

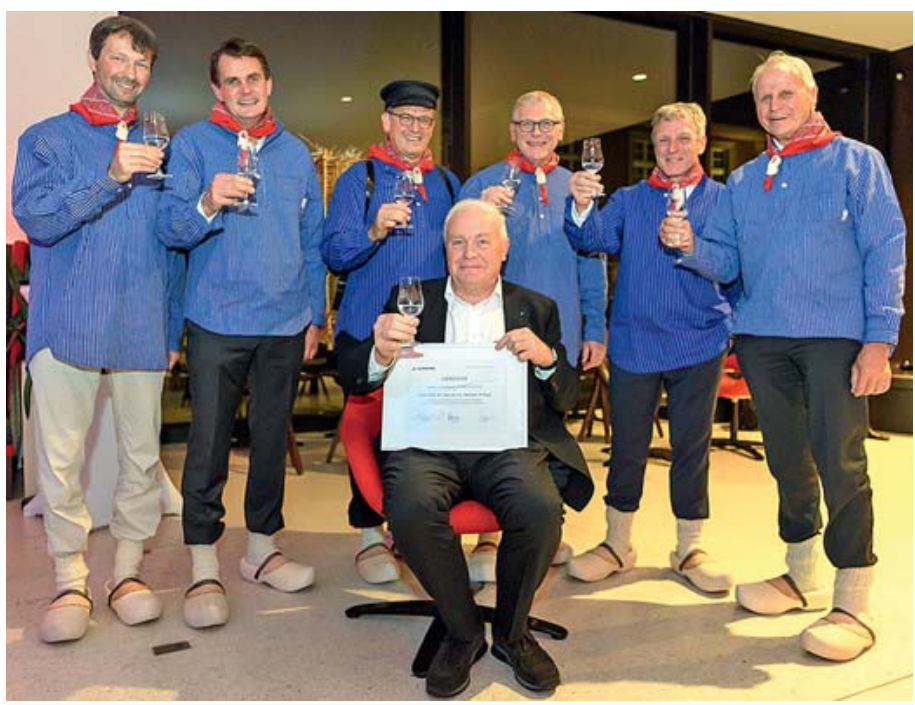

Abb. 5 Ehrung von Haas durch die Kiepenkerle. Bild: AO Foundation.

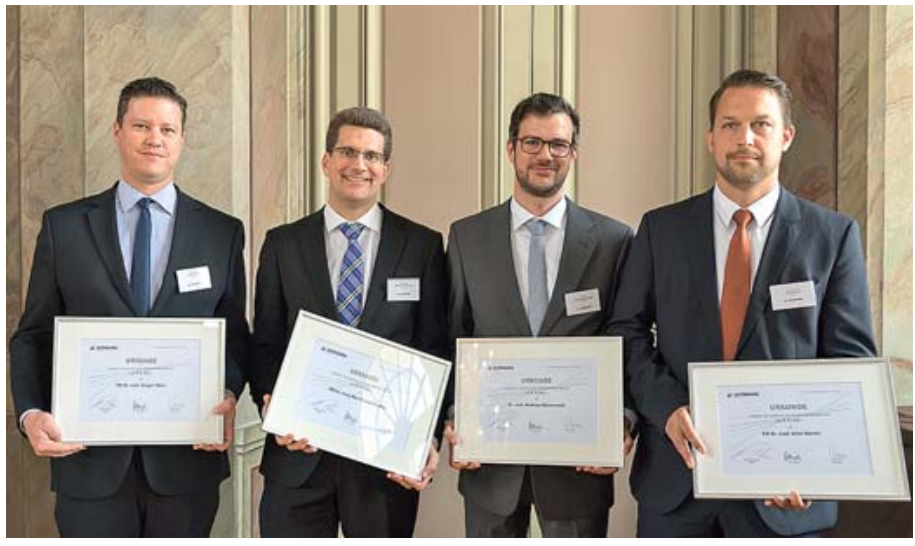

Abb. 6 Stein, Kauther, Baranowski und Berner. Bild: AO Foundation. 


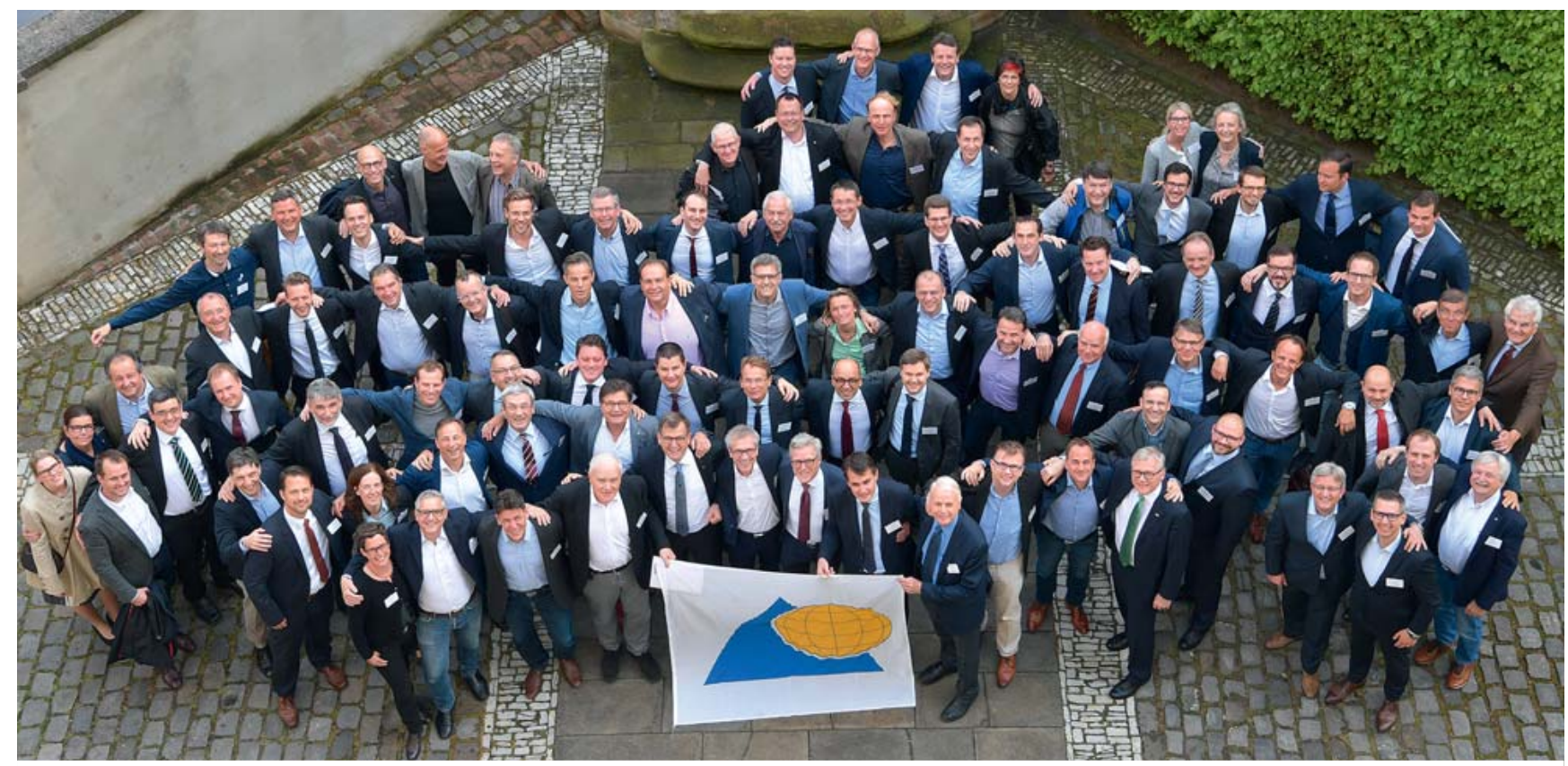

Abb. 7 Gruppenfoto. Bild: AO Foundation.

Nächste Jahrestagung

Die Jahrestagung 2018 findet am 11. und 12.05. in Erfurt in der Klinik von Prof. Mückley statt.

Prof. Dr. Michael J. Raschke, Münster

Philip Wilbrandt, Rechlin

\section{Bibliografie}

DOI https://doi.org/10.1055/s-0043-116394 OP-JOURNAL 2017; 33: 192-194 @ Georg Thieme Verlag KG Stuttgart · New York ISSN 0178-1715 